\title{
Accuracy of the epidural catheter position during the lumbar approach in infants and children: a comparison among L2-3, L3-4, and L4-5 approaches
}

\author{
Yeon A Kim ${ }^{1}$, Ji Young Kim ${ }^{1,2}$, Hae Keum Kil ${ }^{1,2}$, Eun-Mi Kim ${ }^{1}$ Mi Kyeong Kim³ and Hye-sung Kim ${ }^{1}$ \\ ${ }^{1}$ Department of Anesthesiology and Pain Medicine, ${ }^{2}$ Anesthesiology and Pain Research Institute, Yonsei University College of \\ Medicine, ${ }^{3}$ Department of Anesthesiology and Pain Medicine, Kyung Hee University School of Medicine, Seoul, Korea
}

Background: The aim of this study was to compare the accuracy of the position of the epidural catheter inserted from three different lumbar intervertebral spaces, L2-3, L3-4, and L4-5, in infants and children.

Methods: Seventy-five children were randomly allocated to 3 groups according to the epidural catheter insertion site (L2-3, L3-4, and L4-5). The epidural catheter tip was identified using 50\% diluted Iohexol and fluoroscopy. The incidence of correct position was compared among the groups and between infants and children.

Results: The incidence of correct position was significantly higher in the L2-3 group as compared to the L3-4 and L4-5 groups $(\mathrm{P}=0.023$ and $\mathrm{P}=0.046$ respectively). The incidence of correct position was higher in infants compared to children $(\mathrm{P}=0.017)$.

Conclusions: The L2-3 intervertebral space is preferable during epidural catheter insertion in children older than 1 year, but a low lumbar level should be considered in infants because they have a higher risk of neural damage. (Korean J Anesthesiol 2010; 58: 458-463)

Key Words: Epidural analgesia, Pediatrics, Urologic surgery.

\section{Introduction}

Epidural analgesia in infants and children has become possible with the development of pediatric equipment. Its use has been increased due to the increasing demand for postoperative pain control for children. Traditionally, the caudal route was preferred for epidural catheterization in children, but the risk of urofecal infection prevented its placement for postoperative analgesia [1-3]. Alernatively, the thoracic approach allows longer maintenance and has the advantage of reducing the total dose of local anesthetics when the catheter is positioned near the target level of the analgesic dermatome. However, since the thoracic approach requires a greater degree of skill and increases the risk of dural puncture, the lumbar approach has become popular [4]. In the lumbar approach, the catheter must be threaded up or down to the target vertebral level for optimal

Received: November 9, 2009. Revised: 1st, November 23, 2009; 2nd, February 2, 2010. Accepted: March 12, 2010.

Corresponding author: Ji Young Kim, M.D., Department of Anesthesiology and Pain Medicine, Yonsei University College of Medicine, Dogokdong, Gangnam-gu, Seoul 135-270, Korea. Tel: 82-2-2019-3547, Fax: 82-2-2227-7897, E-mail: toughalex@hotmail.com Presented in part at the American Society of Anesthesiologists Annual Meeting, Chicago, October 2006.

(c) This is an open-access article distributed under the terms of the Creative Commons Attribution Non-Commercial License (http:// creativecommons.org/licenses/by-nc/3.0/), which permits unrestricted non-commercial use, distribution, and reproduction in any medium, provided the original work is properly cited. 
analgesia with the least amount of analgesic agent [5-7].

There are no previous studies reporting the accuracy in the desired level of epidural catheter position in pediatric patients. Therefore, we have conducted this study, to assess the accuracy of the position of the epidural catheter as inserted from three different lumbar vertebral spaces, L2-3, L3-4, and L4-5, and we have compared the positions in infants and small children by using fluoroscopic examination with radiopaque dye.

\section{Materials and Methods}

This study was approved by the institutional review board and written informed consent was obtained from the parents of 75 children between the ages of 15 days and 77 months. The children were undergoing urological surgeries having a similar dermatome level of incision. The types of surgeries were pyeloplasy, nephrectomy, and ureteroneocystostomy. Children with general infectious disease, infection focus on the back, coagulopathy, spinal anomaly, bronchial asthma, and drug allergy were excluded from this study. Patients were allocated into three groups according to the epidural catheter insertion site (L2-3, L3-4, and L4-5) using a computer-generated randomization method. An epidural catheter was inserted by two anesthesiologists whose expertise lies in pediatric regional anesthesia.

Anesthesia was induced with sevoflurane, thiopental $5 \mathrm{mg} /$ $\mathrm{kg}$ or propofol $2.5 \mathrm{mg} / \mathrm{kg}$, and rocuronium $0.5 \mathrm{mg} / \mathrm{kg}$. After the tracheal intubation, anesthesia was maintained with sevoflurane (1.5-2.5 vol\%) in air and oxygen. The patients were then placed in lateral position with their backs fully flexed. An ultrasound examination of the lumbar area was performed to evaluate the spinal structure and estimate the epidural depth with a linear probe (LOGIQe, GE Healthcare, USA). After identification of the conus medullaris, the cutaneous distance between the puncture site and the dermatomal level

Table 1. Patient Characteristics

\begin{tabular}{lccc}
\hline & L2-3 $(\mathrm{n}=24)$ & L3-4 $(\mathrm{n}=24)$ & $\mathrm{L} 4-5(\mathrm{n}=24)$ \\
\hline Age (months) & $9.0(0.5-77.0)$ & $11.5(2.0-58.0)$ & $13.5(1.2-54.0)$ \\
Weight (kg) & $9.1(3.9-27.5)$ & $12.55(5.6-23.0)$ & $12.9(4.2-27.0)$ \\
Height (cm) & $81.2(57-116.6)$ & $82.5(63.5-118.3)$ & $83.0(64-126)$ \\
\hline
\end{tabular}

Data are expressed as median (range). of surgical incision was measured. After aseptic preparation, a midline epidural puncture was made with an 18-gauge Tuohy needle (Pelican epidural cannula, B. Braun Medical Inc., Melsungen, Germany). After penetration of the skin and subcutaneous tissues, the needle was advanced in a slightly cephalad direction $\left(75-80^{\circ}\right)$ within the long axis of the vertebral column. The epidural space was identified by the 'drip and tube method' described by Yamashita et al. [8] and the needle was rotated to the direction of the target level. A 20-gauge catheter (Perifix-Paed Soft catheter, B. Braun Medical Inc., Melsungen, Germany) was threaded to the target level according to the previously measured cutaneous distance between the puncture site and the dermatomal level of surgical incision. With the lateral position, 50\% diluted Iohexol (Omnipaque 300, Amersham Health, Cork, Ireland) was injected at a rate of $1 \mathrm{ml}$ over 2 minutes using a syringe pump under cine-fluoroscopy until the distal tip of the epidural catheter was identified within the epidural space [9]. When the distal tip was identified, the radiographic image was taken. Two more images were taken in the lateral and supine position after injection of $0.1 \mathrm{ml} / \mathrm{kg}$ of $50 \%$ diluted Iohexol to examine the dye spread pattern in the epidural space. The images were transferred to picture archiving and communication system (PACS). During fluoroscopy, the patients' genital areas were shielded by a lead plate. To measure the amount of exposure of radiation, we attached a collecting plate to the thigh of 5 randomly selected children during fluoroscopy. The catheter position and dye spread pattern within the epidural space were evaluated with PACS by a radiologist who was blinded to the study group. After the analysis of the puncture site, patients were divided into either infant (birth to 1 year, including 1 year-olds) or children (older than 1 year). The accuracy of catheter position was also compared between infants and children.

Sample size was calculated based on the incidence of correct position in a preliminary study of 32 patients. We expected the incidence of correct position in L 2-3, L3-4, and L4-5 to be $80 \%$, $40 \%$, and $40 \%$ respectively. A sample size of 69 achieved $80 \%$ power to detect an effect size (W) of 0.3780 using a 2 degrees of freedom Chi-square test with a significance level (alpha) of 0.05 . We assumed a dropout rate of $10 \%$ and increased the sample size to 75 patients.

Data were analyzed using SAS (version 6.12, SAS Institute,

Table 2. The Position and Length of the Catheter within the Epidural Space

\begin{tabular}{lcccc}
\hline & L2-3 $(\mathrm{n}=24)$ & L3-4 $(\mathrm{n}=24)$ & L4-5 (n=24) & Total \\
\hline Correct position & $19(79)$ & $10(42)^{*}$ & $12(50)^{*}$ & $41(57)$ \\
Circling & $4(17)$ & $7(29)$ & $9(37.5)$ & $20(28)$ \\
Doubling back & $1(4)$ & $7(29)$ & $3(12.5)$ & $11(15)$ \\
Catheter length $(\mathrm{cm})$ & $3.5(2.8-4.5)$ & $4.0(3.2-7.0)^{*}$ & $4.8(4.2-5.0)^{*, \dagger}$ & \\
\hline
\end{tabular}

Data are expressed as number (percentage) or median (range). *P $<0.05$ compared to value in $\mathrm{L} 2-3$ group, ${ }^{\dagger} \mathrm{P}<0.05$ compared to value in $\mathrm{L} 3-4$. 
Cary, NC, USA). The Kruskal-Wallis test was used for analysis of age, weight, and height. The Kruskal-Wallis test followed by Bonferroni correction was used for catheter length. The differences among the three groups for incidence of correct position were analyzed with a Chi-square test with Fisher's exact test correction. The incidence of correct position between infants and children was analyzed using Fisher's exact test. Values were expressed as median (range) or number of patients (percentage). A P value of less than 0.05 was considered statistically significant.

\section{Results}

A total of 75 patients were randomly allocated into three groups. Among the 25 patients recruited in each group, one
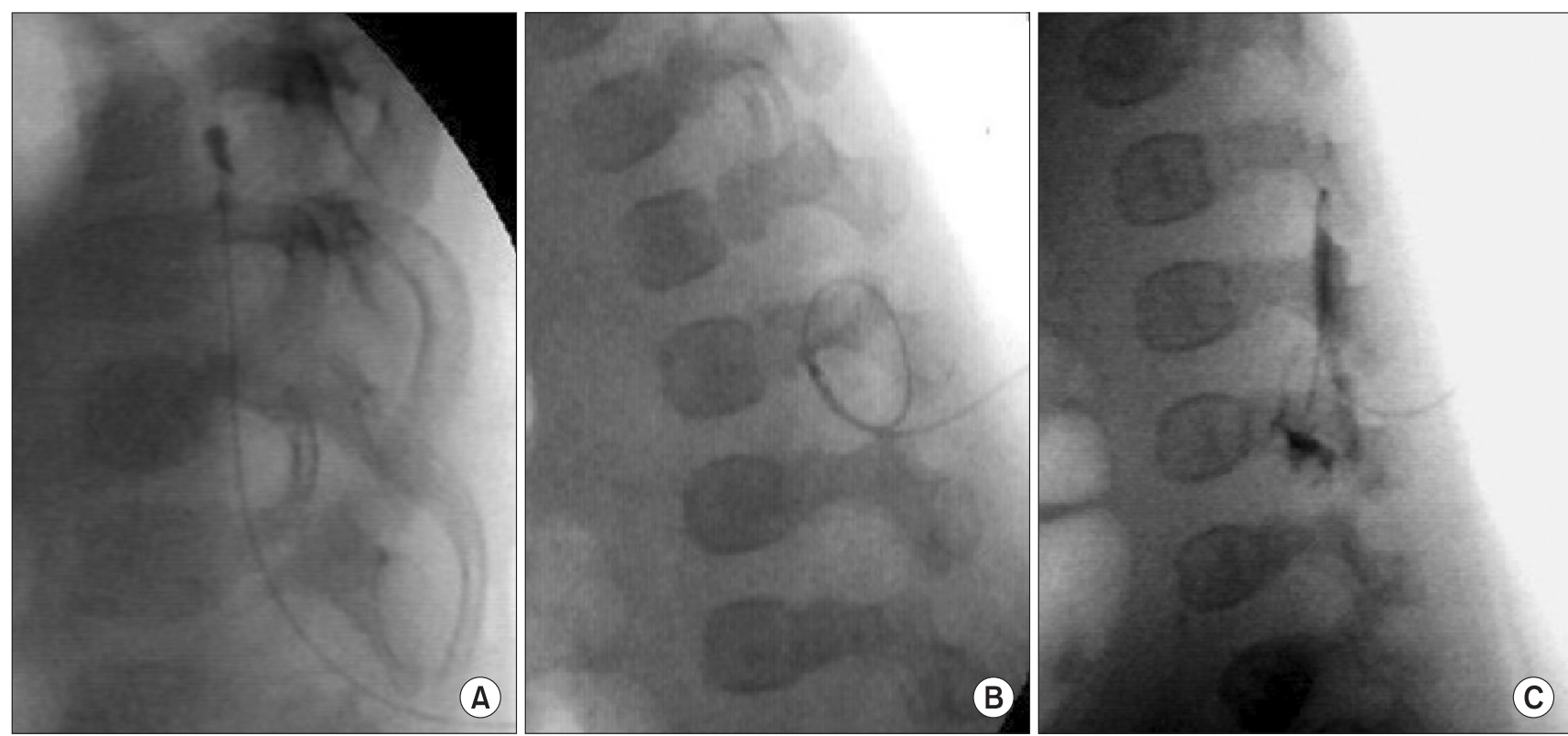

Fig. 1. Various positions of the catheters within the epidural space. (A) Straight-up (B) Circled (C) Turning-back position of catheter.
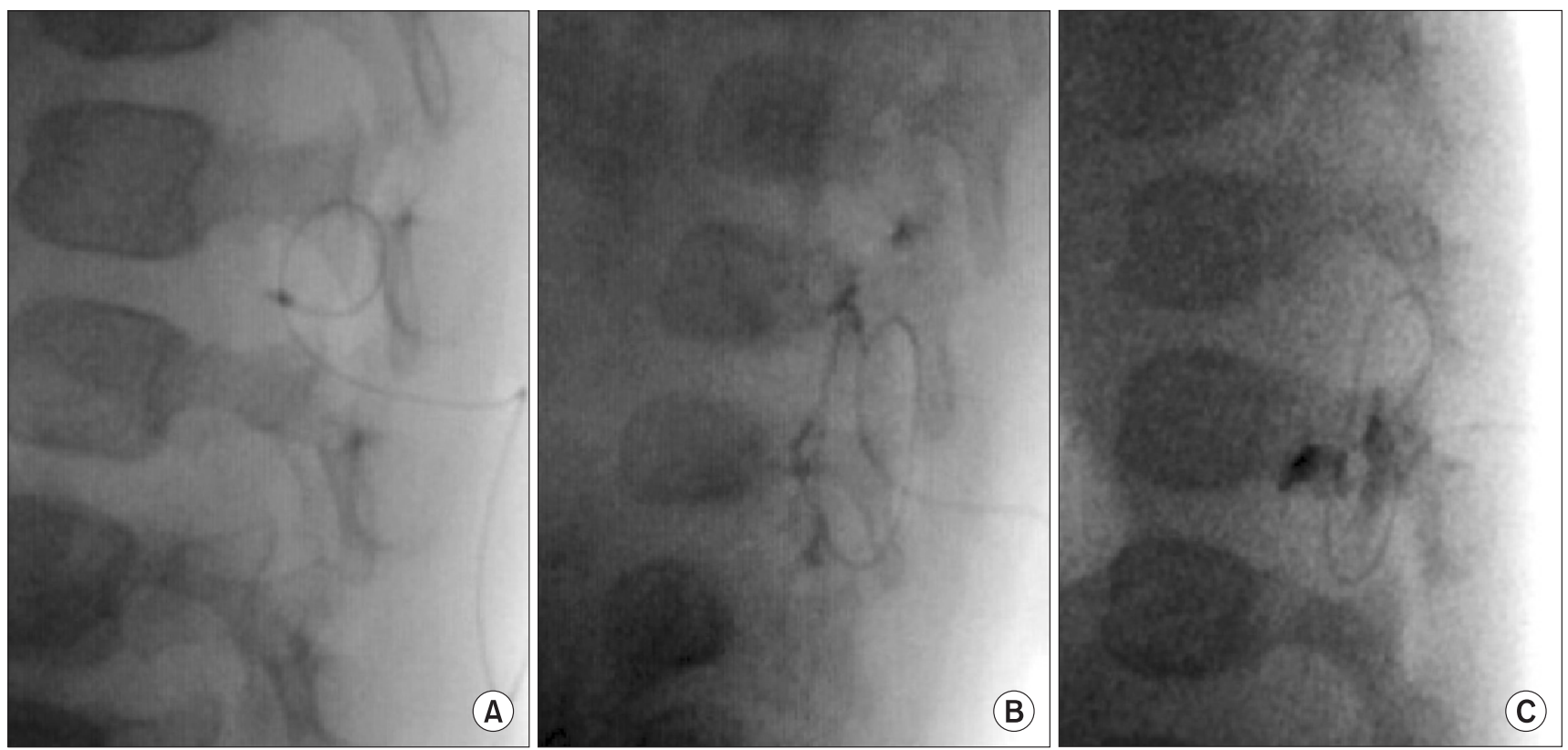

Fig. 2. Patterns of circled catheters within the epidural space. (A) Circling itself above the point of entrance (B) Near the point of entrance (C) Forming a figure of eight. 

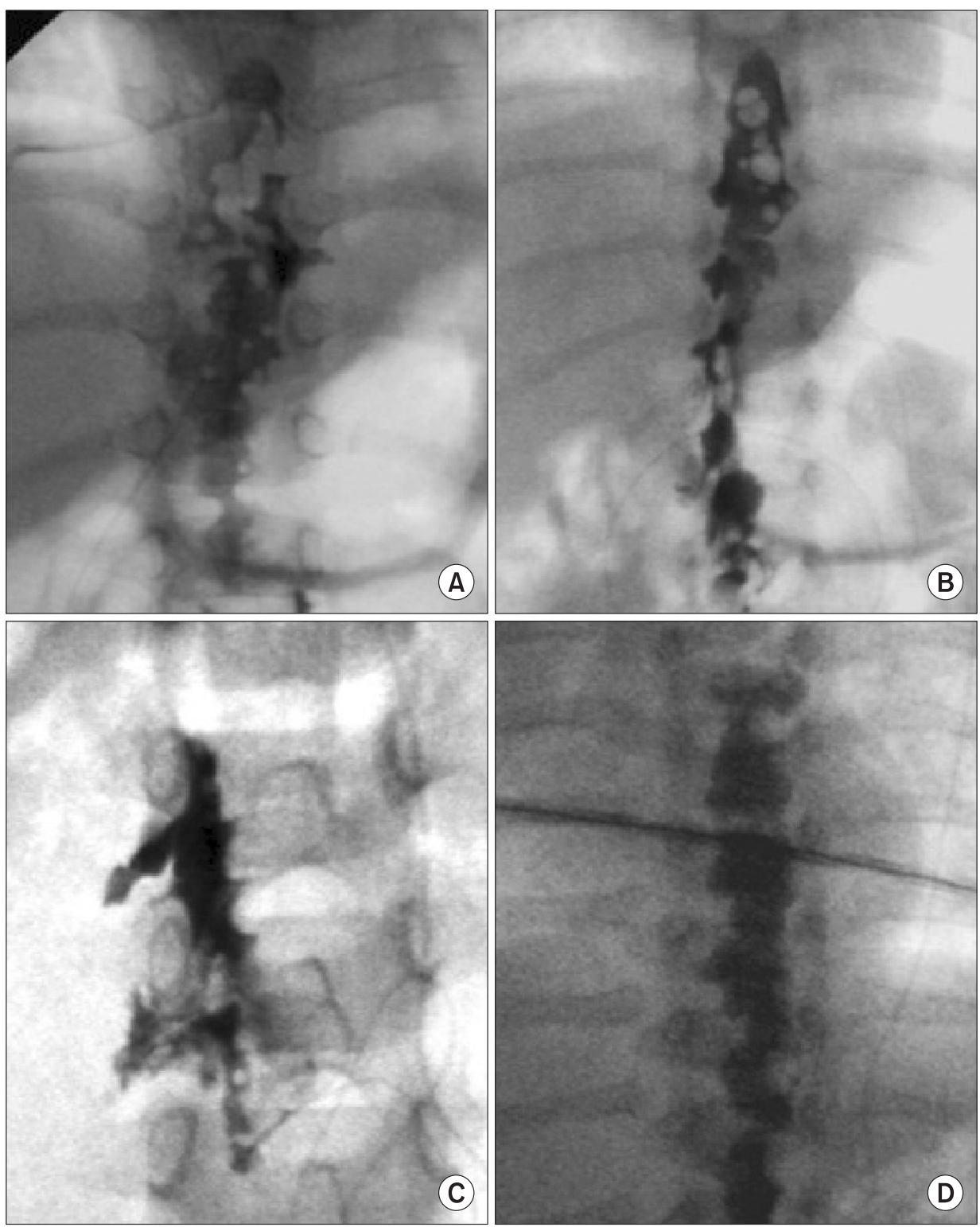

Fig. 3. Patterns of dye spread. (A) Segregated patches (B) Filling defect (C) Paravertebral leaks (D) Central midline spread.

patient in each L2-3 and L3-4 group was excluded from the analysis due to catheter malfunction. One in the L 4-5 group was also excluded from the analysis due to loss of stored images.

Patient characteristics are presented in Table 1. There was no statistical difference in age, weight, and height among the three groups. All punctures were successful on the first attempt and there was no case of dural puncture, bleeding, failure of catheterization or allergic reactions to radiopaque dye.

The position and length of the catheter within the epidural space were listed in Table 2. The catheters traveled straightup, circling, or doubling back in the epidural space (Fig. 1A$\mathrm{C}$, respectively). Catheter traveling straight-up was positioned correctly. The difference in the incidence of correct position was statistically significant $(P=0.041)$, and the incidence was significantly higher in the L2-3 group compared to that in the L3-4 and L4-5 groups ( $\mathrm{P}=0.023$ and $\mathrm{P}=0.046$ respectively). Overall incidence of correct position (catheter traveling straight up) was $41 / 72(0.57,95 \%$ CI $0.45-0.68)$. In 4 of the total 41 straight up cases, the catheter traveled in a wavy line within the epidural space, but the catheter tip reached the target level. Incorrect position occurred in circling and doubling back of the catheter in the epidural space. Circling patterns are shown in Fig. 2. The circling near the point of entrance without traveling further up or down the epidural space occurred in 9/20 (0.43 $95 \%$ CI $0.24-0.63$ ) of the total circling cases. In 2 doubling back cases, the catheter was headed in the opposite direction of the target site and then doubled back to the target direction. The difference in the length of the catheter in the epidural space was 
statistically significant $(\mathrm{P}=0.000)$ and the length was statistically shorter in the L 2-3 group compared to that in the L 3-4 and $\mathrm{L}$ 4-5 groups $(\mathrm{P}=0.001, \mathrm{P}=0.000)$.

The incidence of correct position in infants and children was 28/40 (0.7 95\% CI 0.54-0.82) and 13/32 (0.4 95\% CI 0.25-0.58) respectively. The incidence was higher in infants compared to that in children $(\mathrm{P}=0.017)$.

Dye spread patterns with $0.1 \mathrm{ml} / \mathrm{kg}$ of $50 \%$ diluted Iohexol are shown in Fig. 3. Segregated patches, filling defects, paravertebral leaks, and central midline spread patterns were observed (Fig $3 \mathrm{~A}-\mathrm{D}$, respectively). The average upward and downward spread was $2.0 \pm 0.9$ and $1.7 \pm 1.1$ segments, respectively. The amount of radiation detected in 5 selected cases was $0.05-0.26$ $\mathrm{mSv}$, which was less than the background radiation present in the atmosphere $(1-2 \mathrm{mSv})$.

\section{Discussion}

This study demonstrated that the incidence of correct epidural catheter placement to target level during lumbar approach is the greatest when the L2-3 interspace is used. The incidence of correct positioning was higher in infants compared to that in children older than 1 year.

The epidural catheter in pediatric patients can be placed through the caudal, lumbar, or thoracic approach. The catheter tip should be placed near the surgery level in order to provide an effective analgesia and this is best achieved when the catheter is inserted near the target dermatome. However, a higher risk of neural damage in the thoracic approach [10] and a risk of urofecal infection in the caudal approach [3] caused the lumbar approach to be used widely for postoperative epidural analgesia. During the lumbar approach, advancing the epidural catheter from the lumbar interspace to the target thoracic level often resulted in incorrect positioning of the catheter $[5,11]$. Blanco et al. [5] reported that the catheter reached the expected level in only $22 \%$ of 39 children older than 1 year when it was advanced from the lower lumbar level with a perpendicular midline approach. The low incidence of correct position of the catheter may have resulted from development of the lumbosacral curvature over 1 year of age as a consequence of standing and walking in children over 1 year of age $[1,11]$. The higher incidence of correct position in the L2-3 group in this study may also supports the lumbosacral curvature interferences on catheter advancement.

The higher incidence of correct catheter position in infants in this study may be explained in part with immature tissue development, ossification, and the lumbosacral curvature that was mentioned earlier. In addition, low lumbar intervertebral space is preferred in infants younger than 1 year-old because the cranial migration of the terminal end of the spinal cord from the L3 level at birth to L1-2 is incomplete. Since the incidence of correct catheter position was higher in infants in this study, low lumbar intervertebral space may decrease the risk of spinal cord damage without the risk of increasing catheter malposition in the epidural space in infants.

As with the lumbosacral curvature, the length of catheter advancement is a factor that may influence the accuracy of the epidural catheter placement. As expected, the catheter length was statistically shorter in the L2-3 group compared to other groups. Care should be taken during removal of the epidural catheter because a catheter length longer than $3 \mathrm{~cm}$ may be coiled, with the potential risk of knotting during removal [12-15]. In this study, although the catheter length was longer than $3 \mathrm{~cm}$ even in the L2-3 group, it was removed without any difficulty in all patients.

The total incidence of correct position of the catheter was higher than that previously reported by Blanco et al. [5] (57\% vs. $22 \%$ respectively). The incidence of correct position in children older than 1 year in this study was also higher than that of Bianco et al. ( $41 \%$ vs. $22 \%$ ). This may have resulted from the difference in the insertion angle of the epidural needle. In adult studies, a perpendicular median approach created a $90^{\circ}$ angle between the bevel of the needle and the epidural space. Leeda et al. reported that this made the catheter tip bend at the contact with the dura mater, consequently increasing resistance to the advancement of the catheter $[16,17]$. They suggested a steeper needle angle was the reason for easier catheter insertion with the paramedian approach. Further study is needed to elucidate the relationship between the angle of needle insertion and correct position.

The spread pattern was also evaluated in children in this study. The average upward and downward spread was $2.0 \pm 0.9$ and $1.7 \pm 1.1$ segments, respectively, with $0.1 \mathrm{ml} / \mathrm{kg}$ of bolus dye. The volume of the dye was chosen to identify the spread pattern because the epidural catheter was inserted in children for continuous postoperative analgesia, in which a small volume of the analgesic agents was infused. This spread pattern and short segmental spread emphasized the importance of the correct position of the epidural catheter for optimal postoperative pain control.

In conclusion, the L2-3 lumbar intervertebral space is preferred during epidural catheter insertion in children older than 1 year to increase the incidence of correct catheter position and to reduce the risk of catheter related complications. However, a low lumbar level should be considered in infants because of the higher risk of the neural damage.

\section{References}

1. Bösenberg AT, Bland BA, Schulte-Steinberg O, Downing JW. 
Thoracic epidural anesthesia via caudal route in infants. Anesthesiology 1988; 69: 265-9.

2. Tsui BC, Wagner A, Cave D, Kearney R. Thoracic and lumbar epidural analgesia via the caudal approach using electrical stimulation guidance in pediatric patients: a review of 289 patients. Anesthesiology 2004; 100: 683-9.

3. Zwass MS. Regional anesthesia in children. Anesthesiol Clin North America 2005; 23: 815-35.

4. Soliman LM, Mossad EB. Thoracic epidural catheter in the management of a child with an anterior mediastinal mass: a case report and literature review. Paediatr Anaesth 2006; 16: 200-5.

5. Blanco D, Llamazares J, Rincón R, Ortiz M, Vidal F. Thoracic epidural anesthesia via the lumbar approach in infants and children. Anesthesiology 1996; 84: 1312-6.

6. Peutrell JM, Lonnqvist PA. Neuraxial blocks for anaesthesia and analgesia in children. Curr Opin Anaesthesiol 2003; 16: 461-70.

7. Tiso RL, Thomas PS, Macadaeg K. Epidural catheter direction and local anesthetic dose. Reg Anesth 1993; 18: 308-11.

8. Yamashita M, Tsuji M. Identification of the epidural space in children. The application of a micro-drip infusion set. Anaesthesia 1991; 46: 872-4.

9. Van Niekerk J, Bax-Vermeire BM, Geurts JW, Kramer PP. Epidurography in premature infants. Anaesthesia 1990; 45: 722-5.

10. Kasai T, Yaegashi K, Hirose M, Tanaka Y. Spinal cord injury in a child caused by an accidental dural puncture with a single-shot thoracic epidural needle. Anesth Analg 2003; 96: 65-7.

11. Jylli L, Lundeberg S, Olsson GL. Retrospective evaluation of continuous epidural infusion for postoperative pain in children. Acta Anaesthesiol Scand 2002; 46: 654-9.

12. Valairucha S, Seefelder C, Houck CS. Thoracic epidural catheters placed by the caudal route in infants: the importance of radiographic confirmation. Paediatr Anaesth 2002; 12: 424-8.

13. Beilin Y, Bernstein HH, Zucker-Pinchoff B. The optimal distance that a multiorifice epidural catheter should be threaded into the epidural space. Anesth Analg 1995; 81: 301-4.

14. Brichant JF, Bonhomme V, Hans P. On knots in epidural catheters: a case report and a review of the literature. Int J Obstet Anesth 2006; 15: 159-62.

15. Lim YJ, Bahk JH, Ahn WS, Lee SC. Coiling of lumbar epidural catheters. Acta Anaesthesiol Scand 2002; 46: 603-6.

16. Leeda M, Stienstra R, Arbous MS, Dahan A, Th Veering B, Burm AG, et al. Lumbar epidural catheter insertion: the midline vs. the paramedian approach. Eur J Anaesthesiol 2005; 22: 839-42.

17. Blomberg RG. Technical advantages of the paramedian approach for lumbar epidural puncture and catheter introduction. A study using epiduroscopy in autopsy subjects. Anaesthesia 1988; 43: 83743. 